\title{
美捷登点评2016年（2015年度）影响因子
}

\section{- - 系统分析开放获取的SCIE及SSCI期刊}

\author{
Dr. Mike Wang
}

背景

开放获取 (Open Access, OA) 期刊就是那些向作者免费开放其 同行评议的文章的期刊。根据期 刊开放获取程度及时间, 大致分 为以下三类开放获取期刊 : 完全 开放获取期刊 (full open access journals)、混合型开放获取期刊 (hybrid open access journals) 和 延迟开放获取期刊 (delayed open access journals)。

与完全开放获取期刊 (所有 文章都向读者立即开放获取 ) 相 比, 混合型开放获取期刊的部分 文章可以开放获取, 而其余文章 还是读者通过传统的订阅方式来 获取; 而延迟开放获取期刊的文 章仅在特定时期内对订阅读者开 放, 而在特定时期 (通常为 12-24 个月）后自动对所有读者开放。

OA 期刊的文章主要通过金 色通道 (Gold road, 也称为 Gold OA, 即期刊官网) 和绿色通道 （Green road, 或 Green OA）实 现开放获取。金色通道是开放获 取期刊通过自己的官网来实现的, 而绿色通道是通过把文章自存档 于机构知识库 (Institutional Repositories ; 比如哈佛大学学术库
DASH) 或学科知识库 (Disciplinary Repositories ; 比如 https:// arxiv.org/, 收集物理、数学、计 算机和生物学非同行评议论文) 中来实现。本文主要对通过金色 通道实现开放获取的 OA 期刊进 行分析讨论。

由于各大出版商旗下的 $\mathrm{OA}$ 期刊非常多, 比如 Elsevier 出 版集团旗下就有 549 份 OA 杂志 (https://www.elsevier.com/about/ open-science/open-access/openaccess-journals)、而所有 OA 杂志 (包括 SCI 和非 SCI 的)有近万份, 所以本文只对汤森路透集团旗下 的 Web of Science 上的所收录的 科学版 SCIE 和社会科学版 SSCI $\mathrm{OA}$ 杂志进行分析。以下提到的 OA 期刊均为 SCIE 或 SSCI 收录
的。

一、OA期刊数量逐年增长

笔者收集并分析了从 20012015 的 15 年的 SCIE 和 SSCI 类 OA 期刊的数据: SCIE 的 OA 期 刊数量从 2001 年的 154 份逐年 上升, 其中以 2009 和 2010 两年 的增加量最多, 分别增加了 190 和 188 份期刊, 上升到 2015 年的 1069 份; 同样, SSCI 的 OA 期 刊数量也从 2001 年的 19 份开始 逐年上升, 同样是 2009 和 2010 两年的增加量最多, 分别增加了 48 和 40 份期刊, 上升到 2015 年 的 184 份。

2015 年 SCIE 和 SSCI 类期 刊总数量比 2001 年的期刊数量

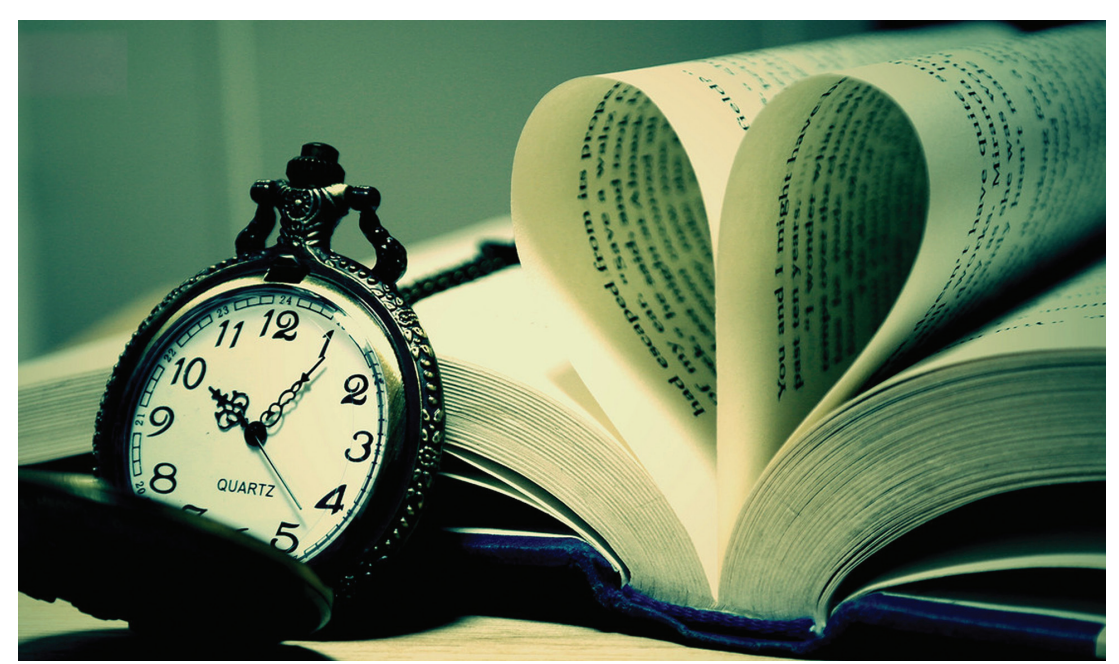




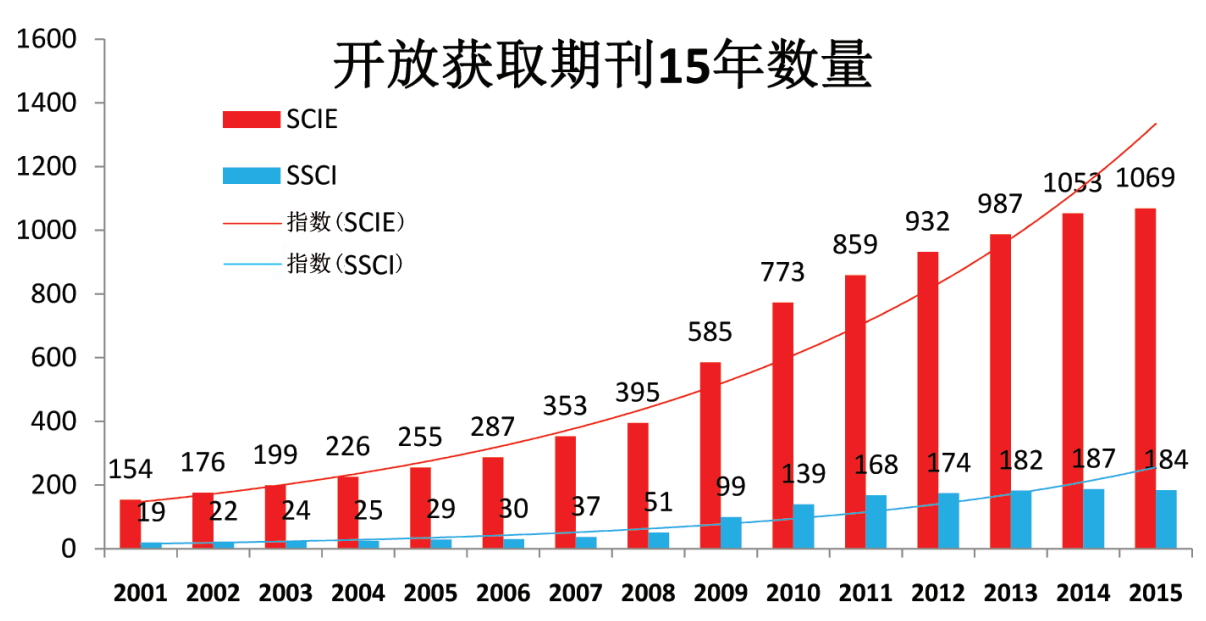

图1.2001-2015自然科学和社合科学SCIDOA期刊的数量增长情况

翻了 7 番和 9 番, 而 $\mathrm{OA}$ 的 $\mathrm{SCIE}$ 期刊占所有 SCIE 期刊的比例由 2001 年的 2.68\% (154/5752) 上升 到 2015 年的 $12.18 \%$ (1069/8778)； OA 的 SSCI 期刊占所有 SSCI 期刊的比例由 2001 年的 $1.13 \%$ (19/1682) 上升到 2015 年的 5.73\% (184/3212)（图 1)。由此可见, 现如今 $\mathrm{OA}$ 期刊已成为了一种趋 势。

\section{二、各大出版集团旗下的} OA杂志

\section{BioMed Central (BMC)}

$\mathrm{BMC}$ 是于 2000 年成立的较 早开始运营 OA 期刊的出版商, 并于 2008 年并入 Springer 出版 集团。在其官网上列有 315 份 $\mathrm{OA}$ 杂志, 其中在 2016 年被 Web of Science 数据库收录的有 175 份杂志, 这 175 份中有 158 份是 $\mathrm{OA}$ 期刊。虽然剩余的 17 份杂志 在 BMC 网址上也显示是 $\mathrm{OA}$ 杂 志, 但是没有被 Web of Science
数据库归类为 $\mathrm{OA}$ 期刊, 很有可 能 Web of Science 有其对 OA 杂 志划分的更高标准。此情况也在 其它出版商的杂志中出现。

$\mathrm{BMC}$ 的这 158 份 $\mathrm{OA}$ 期刊 在 2015 年共发表了 23566 篇文 章, 其中 BMC Public Health (1304 篇)、BMC Genomics (1176 篇) 和 BMC Cancer (1019 篇) 发表 量较大, 均超过一千篇, 其影响 因子最高的 OA 类 SCIE 杂志为 Genome Biology, IF : 11.313, 发 表量为 230 篇 (表格 1)。

\section{HINDAWI}

HINDAWI 是于 1997 年成立 的埃及出版集团, 其官网上列有 414 份杂志 OA 杂志, 不过 2015 年被 SCIE 收录的只有 53 份, 其 中 44 份是 OA 的 SCIE 杂志。 HINDAWI 在 2015 年共发表了 14351 篇 OA 文章, 其中 Biomed Research International (3179 篇) 和 Mathematical Problems in Engineering (2260 篇) 发表量超过
两千篇, Evidence-based Complementary and Alternative Medicine 发表量接近一千篇(924 篇)。其 影响因子最高的 OA 类 SCIE 杂志 为 Oxidative Medicine and Cellular Longevity，IF : 4.492，发表 量为 290 篇 (表格 1)。

\section{MDPI (Multidisciplinary Digi- tal Publishing Institute，鸟 学科数字出版机构)}

MDPI 是 2010 年由国际分 子多样性保护组织 (Molecular Diversity Preservation International）更名而来的出版公司。该 出版商旗下多数杂志名为一个 单词。MDPI 官网上列有 161 份 杂志为 $\mathrm{OA}$ 杂志, 不过 2015 年 被 SCIE 收录的 OA 杂志只有 26 份。这 26 份杂志在 2015 年共 发表了 12465 篇 OA 文章, 其中 International Journal of Molecular Sciences (1725 篇)、Sensors (1649 篇) 和 Molecules (1347 篇) 发表量超过一千篇, International 
表1. 各大出版商旗下的 $0 A$ 期刊及其文章信息

\begin{tabular}{|c|c|c|c|}
\hline Publishers & OA/Total & $\begin{array}{l}2015 \text { Total citable } \\
\text { items (OA/Total) }\end{array}$ & OAJ with high amount of papers in 2015 \\
\hline BIOMED CENTRAL & $158 / 175$ & $23566 / 25258$ & $\begin{array}{l}\text { BMC Public Health，IF: 2.209; 1304篇 } \\
\text { BMC Genomics, IF: 3.867; 1176篇 } \\
\text { BMC Cancer, IF: 3.265; 1019篇 }\end{array}$ \\
\hline HINDAWI & $44 / 53$ & $14351 / 15072$ & $\begin{array}{l}\text { Biomed Research International, IF: 2. 134; 3179篇 } \\
\text { Mathematical Problems in Engineering, IF: } 0.644 ; 2260 \\
\text { 篇 } \\
\text { Evidence-based Complementary and Alternative } \\
\text { Medicine, IF: 1.931; 924篇 }\end{array}$ \\
\hline MDPI & $26 / 27$ & $12465 / 12605$ & $\begin{array}{l}\text { International Journal of Molecular } \\
\text { Sciences, IF: 3.257; } 1725 \text { 篇 } \\
\text { Sensors, IF: 2.033; 1649篇 } \\
\text { Molecules, IF: 2.465; } 1347 \text { 篇 } \\
\text { International Journal of Environmental Research } \\
\text { and Public Health, IF: 2.035; 984篇 }\end{array}$ \\
\hline $\begin{array}{l}\text { FRONTIERS } \\
\text { MEDIA S. A }\end{array}$ & $16 / 18$ & $6377 / 6701$ & $\begin{array}{l}\text { Frontiers in Microbiology, IF: 4. 165; 1, 435篇 } \\
\text { Frontiers in Plant Science, IF: 4.495; 1, 135篇 } \\
\text { Frontiers in Immunology, IF: 5.695; 559篇 }\end{array}$ \\
\hline $\begin{array}{l}\text { WILEY- } \\
\text { BLACKWELL }\end{array}$ & $15 / 775$ & $2263 / 98697$ & $\begin{array}{l}\text { Ecology and Evolution, IF: 2.537; 495篇 } \\
\text { Molecular Systems Biology, IF: 10.581; 64篇 } \\
\text { EMBO Molecular Medicine, IF: 9.547; 98篇 } \\
\text { Aging Cell, IF: 5. 760; 115篇 } \\
\text { Earths Future, IF: 5.620; 21篇 } \\
\text { Journal of the American Heart } \\
\text { Association, IF: 5. 117; 373篇 }\end{array}$ \\
\hline ELSEVIER & $13 / 613$ & $2048 / 155411$ & $\begin{array}{l}\text { Physics Letters B, IF: 4.787; 826篇 } \\
\text { Redox Biology, IF: 6. 235; 143篇 } \\
\text { Lancet Global Health, IF: 14.722; 57篇 } \\
\text { Materials Today, IF: 17.793; 40篇 }\end{array}$ \\
\hline $\begin{array}{l}\text { NATURE } \\
\text { PUBLISHING } \\
\text { GROUP }\end{array}$ & $13 / 90$ & $11623 / 26155$ & $\begin{array}{l}\text { Translational Psychiatry, IF: 5.538; 203篇 } \\
\text { Cell Death \& Disease, IF: 5. 378; 354篇 } \\
\text { Scientific Reports, IF: 5.228; 10642篇Experimental } \\
\text { \& Molecular Medicine, IF: 5. 164; 69篇 } \\
\text { Molecular Therapy-Nucleic Acids, IF: 5.048; 54篇 } \\
\text { Oncogenesis, IF: 5.021; 44篇 }\end{array}$ \\
\hline $\begin{array}{l}\text { PUBLIC LIBRARY } \\
\text { of SCIENCE }\end{array}$ & $7 / 7$ & $31243 / 31243$ & PLoS One, IF: 3.057; 28114篇 \\
\hline $\begin{array}{l}\text { TAYLOR \& } \\
\text { FRANCIS } \\
\text { INC/LTD }\end{array}$ & $5 / 541$ & $270 / 47021$ & Acta Orthopaedica, IF: 2.617; 116篇 \\
\hline SPRINGER & $4 / 683$ & $573 / 91886$ & Nanoscale Research Letters, IF: 2.584；470篇 \\
\hline $\begin{array}{l}\text { SAGE } \\
\text { PUBLICATIONS }\end{array}$ & $4 / 193$ & $762 / 17084$ & Advances in Mechanical Engineering, IF: 0.640; 537篇 \\
\hline CELL PRESS & $2 / 17$ & $997 / 4688$ & Cell Reports， IF: 7.870; 804篇 \\
\hline $\begin{array}{l}\text { LIPPINCOTT } \\
\text { WILLIAMS \& } \\
\text { WILKINS }\end{array}$ & $1 / 198$ & $1989 / 27387$ & Medicine, IF: 1.206; 1989篇 \\
\hline $\begin{array}{l}\text { CAMBRIDGE } \\
\text { UNIV PRESS }\end{array}$ & $0 / 101$ & $0 / 8680$ & NA \\
\hline
\end{tabular}


Journal of Environmental Research and Public Health 发表量 接近一千篇 (984 篇)。其影响因 子最高的 $\mathrm{OA}$ 类 SCIE 杂志为 $\mathrm{Nu}$ trients, IF : 3.759 , 发表量为 617 篇(表格 1)。

\section{FRONTIERS MEDIA S.A}

Frontiers Media S.A 是 于 2007 年成立的瑞士出版公司。其 旗下有 62 份杂志, 其中有 18 份 被 SCIE 收录, 16 份属于 OA 类 SCIE。这 16 份 OA 期刊 2015 年共发表了 6377 篇文章, 其中 两份杂志发表量过千, 分别是 Frontiers in Microbiology (1,435 篇) 和 Frontiers in Plant Science (1,135 篇)。在这 16 份杂志中, Frontiers in Immunology(559 篇) 的影响因子最高, 为 5.695 (表格 1)。

\section{WILEY-BLACKWELL}

WILEY-BLACKWELL 出版 商旗下的杂志非常多, 其官网显 示为 2379 份杂志, 该出版商旗下 的杂志绝大多数为传统的非 $\mathrm{OA}$ 类期刊, 其中有 775 份被 SCIE 收录。只有 14 份是 $\mathrm{OA}$ 类的 SCIE 杂志, 这 14 份杂志在 2015 年共发表了 2263 篇 OA 文章, 其 中 Ecology and Evolution (495 篇) 发表量最多。其影响因子较高的 OA 类 SCIE 杂志有:Molecular Systems Biology (64 篇), IF :
10.581 EMBO Molecular Medicine (98 篇), IF : 9.547 ;Aging Cell 115 篇), IF : 5.760 ;Earths Future (21 篇), IF : 5.620 ; Journal of the American Heart Association ( 373 篇), IF : 5.117。可见 WILEYBLACKWELL 走的是 “少而精” 的路线 (表格 1)。

\section{ELSEVIER}

ELSEVIER 出版集团旗下有 600 份杂志是非 OA 的 SCIE 期刊, 其旗下共创办了 549 份 OA 期刊, 但是只有 13 份被收录为 SCIE 的 OA 期刊。在这 13 份 SCIE 的 $\mathrm{OA}$ 期刊中, 以 Materials Today (40 篇) 杂志的影响因子最高, 为 17.793 , 其次是 Lancet Global Health (57 篇), 为 14.722 ; 另外 Redox Biology (143 篇) 杂志影 响因子为 6.235。2015 年这 13 份 OA 期刊共发表了 2048 篇文章, 其中Physics Letters B 杂志发表 量最多为 826 篇, IF : 4.787 (表 格 1)。

\section{NATURE PUBLISHING GROUP (NPG)}

NPG 出版集团是大家较为 熟悉的, 其旗下有 90 份杂志被 SCIE 收录, 其中 13 份是 OA 的 SCIE 期刊。2015 年共发表 了 11623 篇 OA 文章, 其中绝大 多数是 Scientific Reports 发表 的。影响因子较高的 OA 类 SCIE
杂志为 Translational Psychiatry (203 篇), IF : 5.538 ; Cell Death \& Disease (354 篇), IF : 5.378 ; Scientific Reports (10642 篇), IF : 5.228 ; Experimental \&Molecular Medicine (69 篇), IF : 5.164 ;Molecular TherapyNucleic Acids (54 篇), IF : 5.048 ; Oncogenesis (44 篇), IF : 5.021(表 格 1)。

多说一句: NPG 旗下的 Scientific Reports 很受大家欢迎, 新 一年影响因子为 5.228 , 并不像大 家预期的那样降幅巨大，那是因 为 2015 年影响因子是根据 2013 年和 2014 年文章发表数 (分别为 2484 和 3931 篇）在 2015 年一整 年内的引用数计算得出的。不过, 该杂志在 2015 年发表了 10642 篇 文章, 远超过了 2013 和 2014 两年 发表总量, 如果引用数量没有同 比增长的话, 估计明年的影响因 子会有大幅下滑 ( 图 2)。

\section{PUBLIC LIBRARY of SCIENCE (PLOS)}

美国科学公共图书馆是于 2002 年成立的出版社, 目前旗 下一共有 7 份杂志, 都是 $\mathrm{OA}$ 的 SCIE 期刊。短短十几年的发展, PLoS 旗下的期刊在生物医学领 域越来越具影响力。2016 年, PLoS 的每个期刊影响因子都在 3.0 以上, 其中以 PLoS Medicine 最高 (IF : 13.585), 余下依次 是 PLoS Biology (IF : 8.668), 


\section{Scientific Reports文章数量}

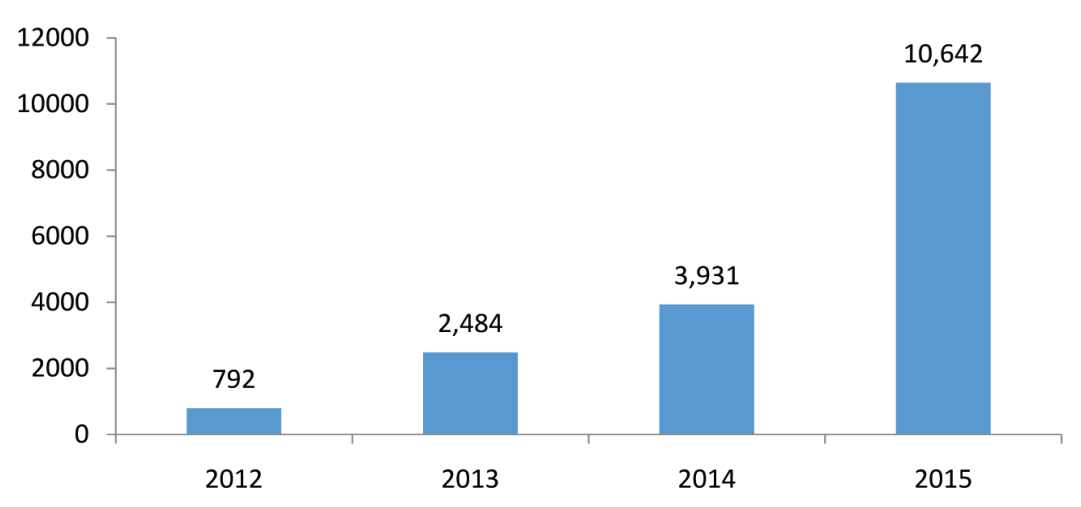

图2. 2002-2015 Scientific Reports期刊文章数量

PLoS Pathogens (IF : 7.003), 和 PLoS Genetics (IF : 6.661)。虽 然 PLoS One (IF : 3.057) 的影响 因子在这 7 份杂志中最低, 但该 杂志是全球上万份杂志中接受发 表论文数量最多的杂志 : 其 2015 年共发表了 28114 篇论文。PLoS
One 杂志的影响因子呈逐年下降 趋势, 而文章发表量在 2013 年 (31,496 篇) 之前呈逐年上升趋 势, 而后逐年下降 (图 3 和 4)。 而 PLoS Medicine 走的是精品路 线, 一年才发表近百篇文章, 其 影响因子最高也不足为奇 (表格 1
和 2)。

\section{TAYLOR \& FRANCIS LTD}

该出版社一共有 541 份杂志 被 SCIE 收录, 其中 5 份杂志为 OA 的 SCIE。这 5 份杂志在 2015

\section{PLoS One IF}

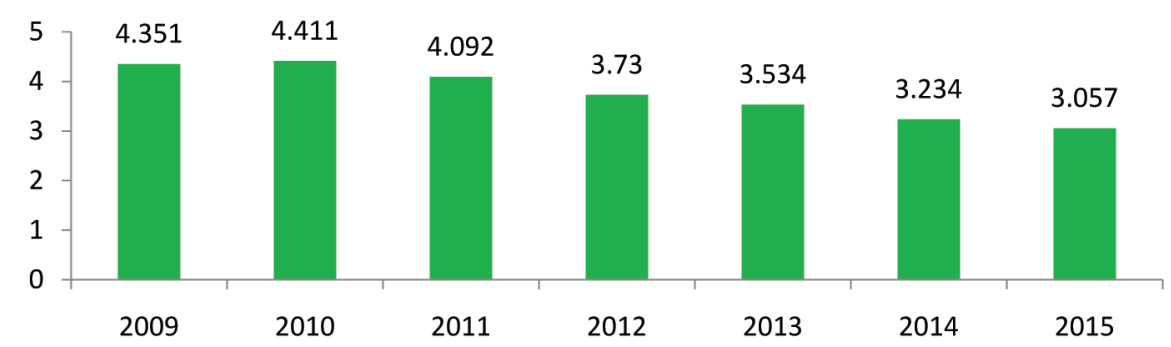

图3. PLoS One 2009-2015影响因子

\section{PLoS One Papers}

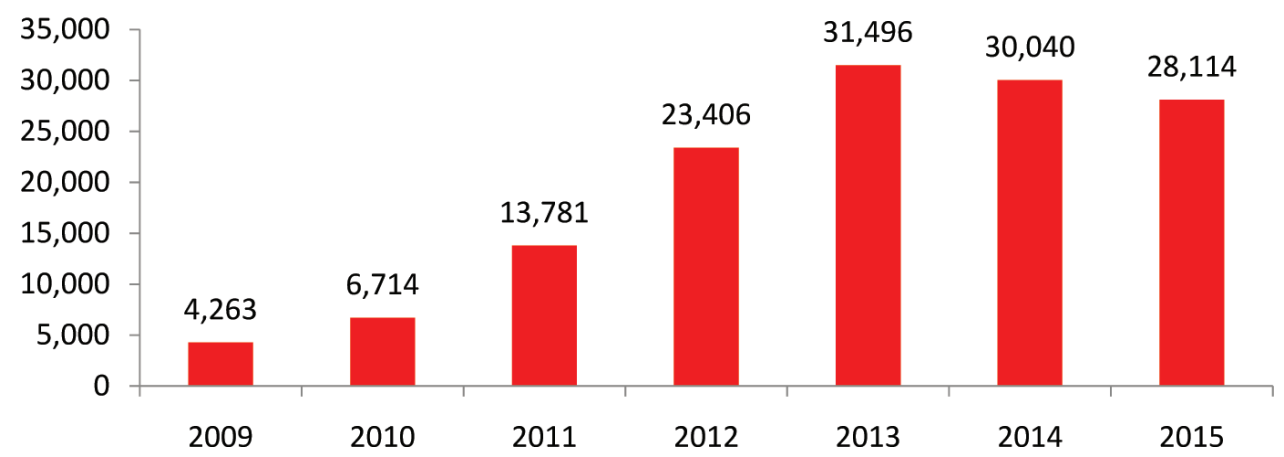

图4.PLoS One 2009-2015发表文章数量 
表格2. PLOS旗TOA期刊信息

\begin{tabular}{llllll}
\hline Rank & Full Journal Title & ISSN & Journal Impact Factor & 2015 papers & Publication Fees \\
\hline 1 & PLoS Medicine & $1549-1676$ & 13.585 & 97 & $\$ 2,900$ \\
2 & PLoS Biology & $1545-7885$ & 8.668 & 183 & $\$ 2,900$ \\
3 & PLoS Pathogens & $1553-7366$ & 7.003 & 693 & $\$ 2,250$ \\
4 & PLoS Genetics & $1553-7404$ & 6.661 & 745 & $\$ 2,250$ \\
5 & PLoS Computational Biology & $1553-734 X$ & 4.587 & 606 & $\$ 2,250$ \\
6 & PLoS Neglected Tropical Diseases & $1935-2735$ & 3.948 & 805 & $\$ 2,250$ \\
7 & PLoS One & $1932-6203$ & 3.057 & 28,114 & $\$ 1,495$ \\
\hline
\end{tabular}

年共发表了 270 篇文章, 其中以 Acta Orthopaedica 杂志发表量 (116 篇) 最多且影响因子最高(IF: 2.617）(表格 1)。

\section{SPRINGER}

SPRINGER 出版集团旗下有 683 份期刊被 SCIE 收录, 其中只 有 4 份为 OA 的 SCIE 期刊。这 4 份 OA 的 SCIE 期刊在 2015 年 发表了 573 篇文章, 其中以 $N a$ noscale Research Letters 发表量 为最多 (470 篇), 该杂志在这 4 份期刊中影响因子也最高, 为 2.584 （表格 1)。

\section{SAGE PUBLICATIONS}

该出版社有 193 份期刊被 SCIE 收录, 其中 4 份属于 OA 的 SCIE 期刊。这 4 份杂志在 2015 年共发表 762 篇文章, 其 中 以 Advances in Mechanical Engineering 发表量最多为 537 篇, IF: 0.640; 而 ASN Neuro (37 篇) 的影响因子最高, 为 2.828 (表格 1)。

\section{CELL PRESS}

该出版社旗下有 17 分杂志被 SCIE 收录, 其中 2 份杂志 (Cell Reports, IF : 7.870 ; Stem Cell Reports, IF : 7.023）属于 $\mathrm{OA}$ 的 SCIE 期 刊。Cell Reports 是 于 2012 年创办的, 过去三年的影响 因子分别为 7.207、8.358 和 7.870, 2015 年该杂志发表了 804 篇文章 (表格1)。

\section{LIPPINCOTT WILLIAMS \& WILKINS}

该出版社旗下有 198 份期刊 被 SCIE 收录, 其中只有 1 份为 OA 的 SCIE 期刊, 即 Medicine 杂志。生物医学领域的研究人员 对此杂志都不陌生, 因为该杂志 的影响因子从去年的 5.723 降到 今年的 1.206 (表格 1)。其下降幅 度在所有 $\mathrm{OA}$ 期刊中为最大 (原 因参看本文后续分析)。

\section{三、OA期刊Top20学科}

在科学版 SCIE 数据库中有 176 类学科领域, 其中 $\mathrm{OA}$ 期刊最
多的前 20 类学科分别为 : MEDICINE, GENERAL \& INTERNAL (49 份) ; NEUROSCIENCES (35 份); PLANT SCIENCES (34 份); PUBLIC, ENVIRONMENTAL \& OCCUPATIONAL HEALTH(33 份 ); MATHEMATICS(31 份); MATERIALS SCIENCE, MULTIDISCIPLINARY(30 份 ); GENETICS \& HEREDITY(29 份); ONCOLOGY (29 份) ; MEDICINE, RESEARCH \& EXPERIMENTAL(28 份) ; GEOSCIENCES, MULTIDISCIPLINARY(28 份 ) ; BIOCHEMISTRY \& MOLECULAR BIOLOGY (27 份); VETERINARY SCIENCES (27 份); ZOOLOGY (25 份); CELL BIOLOGY(24 份) ; PHARMACOLOGY \& PHARMACY(23 份) ; BIOTECHNOLOGY \& APPLIED MICROBIOLOGY(23 份); CHEMISTRY, MULTIDISCIPLINARY (22 份 ) ; METEOROLOGY \& ATMOSPHERIC SCIENCES (20 份) ; IMMUNOLOGY(19 份); BIOLOGY (19 份) (图 5)。 Top20 学科的 OA 期刊一共有 555 


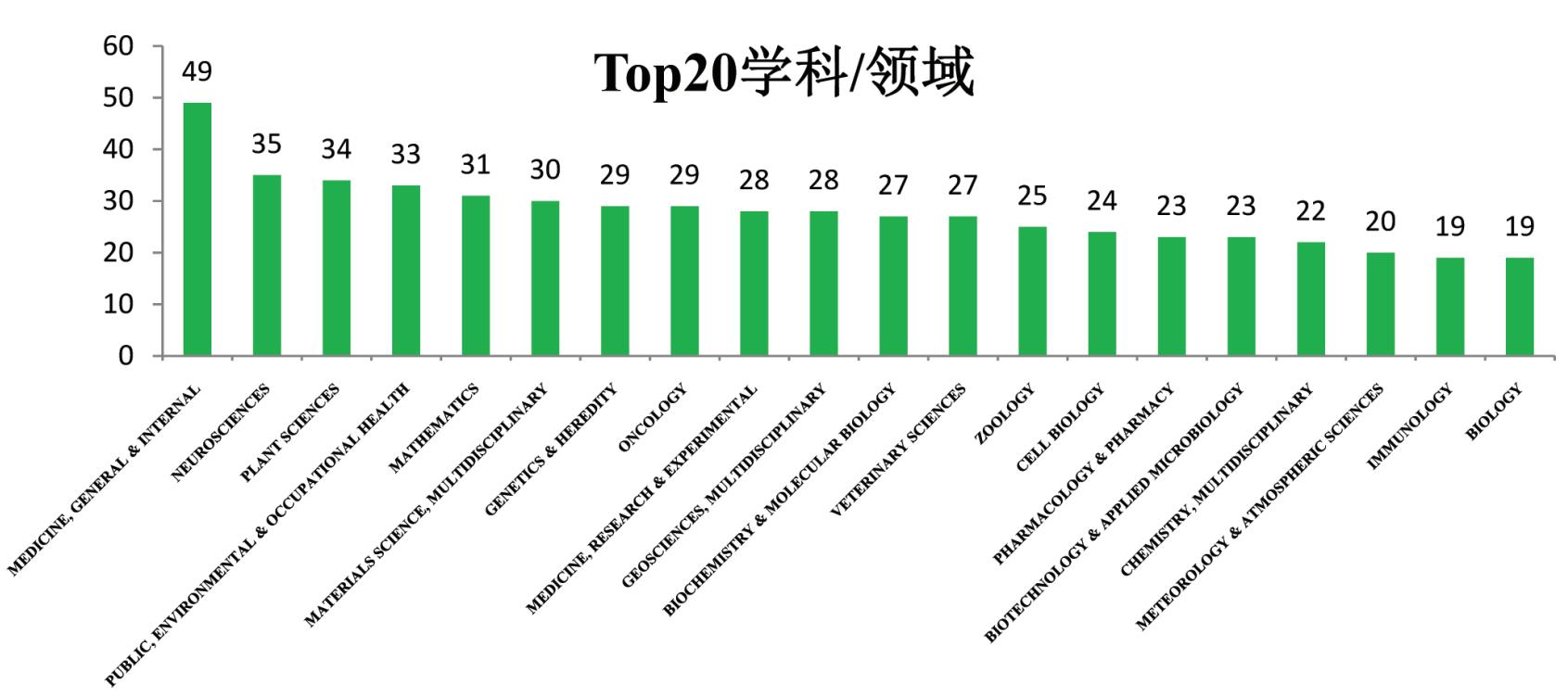

\section{图5. Top20学科/领域期刊数量}

份, 占所有 $\mathrm{OA}$ 期刊的 $52 \%$ 。

四、32份“新晋” 及16

份“被踢出”的0A期刊

在科学版 1069 份 OA 期刊 中, 有 32 份属于新收录的 $\mathrm{OA}$ 杂 志。它们分别为:Morbidity and Mortality Weekly Report; Earth System Science Data ; GigaScience ; Redox Biology ; Frontiers in Immunology; Earths Future ; Skeletal Muscle;Clinical and Translational Gastroenterology; IUCrJ; International Journal of Health Geographics ; $A M B$ Express ; Crystals ; Earth Surface Dynamics ; MycoKeys ; EJNMMI Research; Frontiers in Neurorobotics ; Standards in Genomic Sciences ; Sexual Medicine ; Turkish Journal of Botany; Nature Conservation-Bulgaria ; Geosci- entific Instrumentation, Methods and Data Systems ; International Journal of Agricultural and Biological Engineering;3 Biotech; SpringerPlus ; CytoJournal ; International Journal of Disaster Risk Science ; Videosurgery and Other Miniinvasive Techniques; Pakistan Veterinary Journal ;Emirates Journal of Food and Agriculture ; Acta Amazonica; Phyllomedusa ; The Journal of Applied Research in Veterinary Medicine (图 6)。

这 32 份期刊中，有的是刚被 SCIE 收录的新杂志, 今年刚有第 一年影响因子 ; 有的则是之前被 踢出、去年又被重新收录的。

\section{其中新杂志为（按影响因子 从高到低排序)}

Morbidity and Mortality Weekly Report (MMWR-MORBID
MORTAL $W$ ), IF $: 10.588$, 其 2015 年的文章发表量为 278 篇。 此刊是美国疾病控制中心创办的 杂志, 刚被收录就有这么高的影 响因子, 其原因或许是因为其发 表的文章都是行业领域内系统性 的一年或多年的数据报告, 所以 引用比较多。

Earth System Science Data, IF : 8.286, 是德国 COPERNICUS 出版社旗下的杂志。

GigaScience, IF : 7.463, 属 $\mathrm{BMC}$ 旗下。

Redox Biology, IF : 6.235, 属 ELSEVIER 旗下。

Frontiers in Immunology, IF : 5.695, 属 FRONTIERS MEDIA S.A 出版社旗下。其 2014 年 和 2015 年两年, 每年发表量都在 550 篇左右。

Earths Future, IF : 5.62, 属 WILEY-BLACKWELL 旗下。 该杂志 2015 年只发表 21 篇文章, 


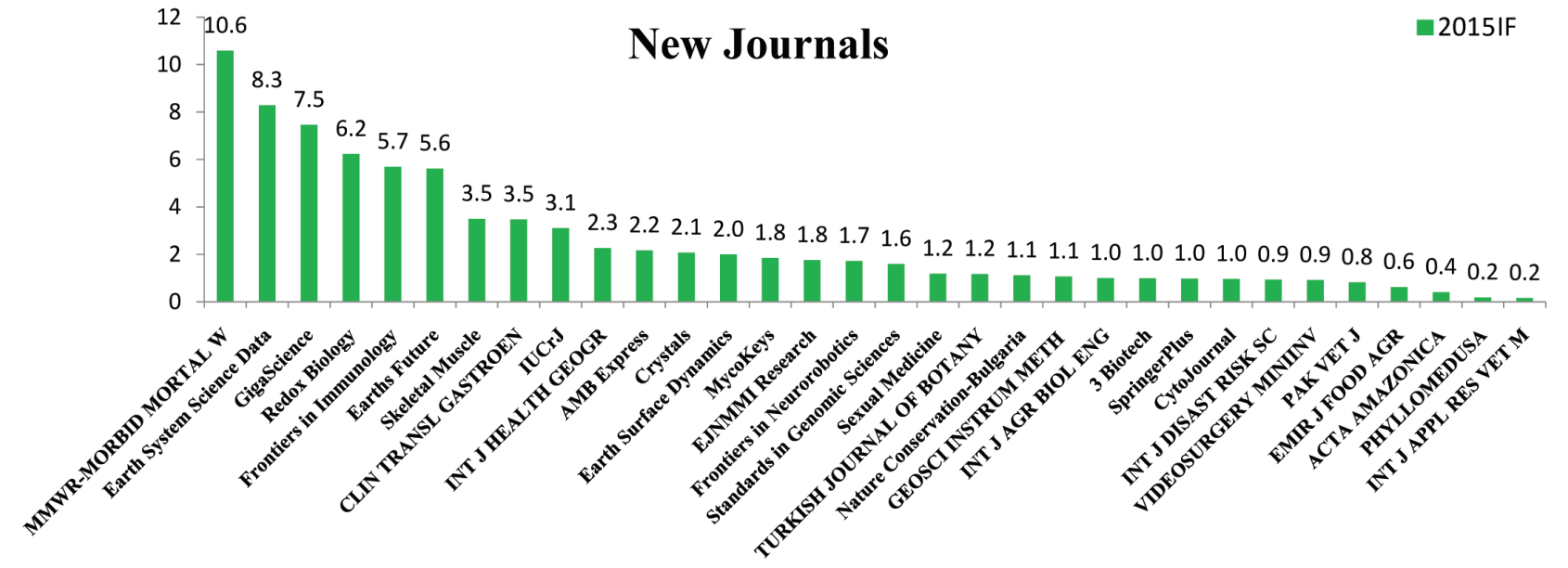

图6.2016年新收录的OA类SCIE期刊

走 “精品” 路线。

Skeletal Muscle, IF : 3.5, 是 BMC 旗下 2011 年创办的杂志, 今年刚被收录为 SCIE 期刊。

Clinical and Translational Gastroenterology (CLIN TRANSL GASTROEN), IF : 3.472, 是 NPG 旗下收录的胃肠道领域 OA 期刊, 过去几年中每年文章发表 量二三十篇。

$I U C r J$, IF : 3.105, 是国际 晶体学联合会 (International Union of Crystallography, IUCr) 旗 下的杂志, 该杂志是以协会英文 缩写词命名的。2014 和 2015 年分 别发表了 57 和 59 篇文章。

International Journal of Health Geographics (INT J HEALTH GEOGR), IF : 2.27, 是 $\mathrm{BMC}$ 旗下杂志, 该杂志创办 了十来年, 是首次被 SCIE 收录, 每年发表大约五六十篇文章。

AMB Express, IF : 2.167, 是 BMC 旗下 2011 年创办的期刊, 首次有影响因子。
Crystals, IF : 2.075, 是 MD PI AG 旗下 2011 年创办的杂志。

Earth Surface Dynamics, IF : 2.000, 是 Copernicus 旗下 2013 年创办的杂志。

MycoKeys, IF : 1.846, 是 PENSOFT 出版社旗下的杂志, 2015 年才发表 10 篇文章。

EJNMMI Research, IF : 1.761, 是 SPRINGER 于 2011 年 创办的杂志, 主要发表核医学和 分子成像领域方面的研究文章。

Frontiers in Neurorobotics, IF $: 1.723$, 是 FRONTIERS MEDIA S.A 出版社旗下的期刊。

Sexual Medicine, IF : 1.188, 是 WILEY-BLACKWELL 旗 下 2013 年创办的期刊。

Nature Conservation-Bulgaria, IF : 1.12, 是 PENSOFT 旗 下 2012 年创办的杂志, 每年发表 十来篇文章。

Geoscientific Instrumentation, Methods and Data Systems (GEOSCI INSTRUM METH),
IF : 1.071, 是 COPERNICUS 旗 下 2012 年创办的杂志。

International Journal of Agricultural and Biological Engineering (INT J AGR BIOL ENG), IF : 1.007 , 是中国农业工程协会 于 2008 年创办的杂志。该杂志今 年用于计算影响因子的引用中有 $52 \%$ 来自于自引, 这么高的自引率, 有一定隐患。

3 Biotech, IF : 0.992, 是 SPRINGER 于 2011 年创办的杂 志。此杂志名很有意思, 意为接 受生物医学、农业和环境三大领 域内的生物技术研究的文章。

SpringerPlus, IF : 0.982, 是 SPRINGER 在 2012 年创办的 期刊, 今年刚刚被收录 SCIE, 并有第一年影响因子。但是就在 今年影响因子发布当日, 该杂志 宣布不再接受新的稿件。笔者猜 想, 或许因为 SPRINGER 去年收 购 NPG 集团以后, 他们已经有了 Scientific Reports, 不想再重复办 类似的箩筐期刊。 
International Journal of Disaster Risk Science (INT J DISAST RISK SC), IF : 0.935 , 是 SPRINGER 旗下的期刊。

Emirates Journal of Food and Agriculture (EMIR J FOOD $A G R)$, IF $: 0.623$, 是 UNITED ARAB EMIRATES UNIV 旗下 的杂志。

Acta Amazonica, IF : 0.408, 和 Phyllomedusa, IF : 0.185 都是 巴西的杂志。

\section{而被重新收录的杂志为（按 影响因子从高到低排序）}

Standards in Genomic Sciences, IF : 1.594, 2014 年之前由 GENOMIC STAND CONSORT 出版社运营, 由于自引率高达 $50 \%$, 导致在 2015 年被 “踢出”, 今年又重新被收录到 SCIE, 目前 归到 BMC 旗下。

Turkish Journal of Botany, IF : 1.178 , 是 TUBITAK SCIENTIFIC \& TECHNICAL RESEARCH COUNCIL TURKEY 主办的, 成立于 1996 年。 2011 年 被 SCIE 收录, 由于自引率超过 $50 \%$ 以上, 2014 年被踢出 SCIE 数据库, 时隔两年后, 今年又重 新被收录到 SCIE 数据库。

CytoJournal, IF : 0.962, 是 MEDKNOW 出版社旗下 2004 年 创刊的杂志, 于 2011 年被 SCIE 收录, 2014 年被踢出 SCIE 数据 库, 今年被再次收录。

Videosurgery and Other
Miniinvasive Techniques (VIDEOSURGERY MINIINV), IF : 0.92, 是 TERMEDIA 出版社旗下的期 刊。该杂志 2011 年被 SCIE 收录, 2015 年被踢出后, 今年又被再次 收录。2014 年的影响因子报告显 示此杂志自引率高达 $80 \%$, 很有 可能是这一原因导致其在去年被 踢出。

Pakistan Veterinary Journal (PAK VET J), IF : 0.822, 1997 年创办, 2010 年被 SCIE 收录, 2015 年被 “踢出” SCIE 数据库, 今年又被再次收录。

International Journal of Applied Research in Veterinary Medicine (INT J APPL RES VET $M)$, IF : 0.159 , 是 VETERINARY SOLUTIONS LLC 旗下杂 志, 于 2009 年被 SCIE 收录，去 年被踢出, 今年又被再次收录。

\section{6年㿭SCIE “踢出”收录 的期刊}

被 “踢出” 的 16 份期刊中有 的是由于改名, 比如 Hindawi 旗 下的两个杂志 : Experimental Diabetes Research 已于 2013 年改 为 Journal of Diabetes Research (IF : 2.431) ; Journal of Biomedicine and Biotechnology 也是于 2013 年改名为 BioMed Research International (IF : 2.134)。其它 改名的杂志还有: Progress of Theoretical Physics (PROG THEOR PHYS) 于 2013 年改名为 Progress of Theoretical and Ex- perimental Physics(IF : 1.889)(图 7)。

另外一些杂志或许是由于自 引率过高被 “踢出”, 比如 Journal of Physical Therapy Science ( $\mathrm{J}$ PHYS THER SCI)、Metalurgija, 以及 SPRINGER 旗下的 Fixed Point Theory and Applications (图 7), 去年影响因子报告中显示其 自引率分别高达 $71 \%$ 、53\% 和 $36 \%$ 。

五、SCIE的OA期刊Top20
分析

去年 SCIE 中 Top20 的 OA 期刊中, 有 16 份仍然在今年的 Top20 中, 它们是: Living Reviews in Relativity (IF : 32)、Living Reviews in Solar Physics (IF : 19.333)、Materials Today (IF : 17.793)、Lancet Global Health (IF : 14.722)、Studies in Mycology (IF : 13.889)、Light-Science \& Applications (IF : 13.6)、PLoS Medicine (IF : 13.585)、Genome Biology (IF : 11.313)、Molecular Systems Biology (IF : 10.581)、 EMBO Molecular Medicine (IF : 9.547) Nucleic Acids Researck IF : 9.202)、Theranostics (IF : 8.854)、 Physical Review X (IF : 8.701)、 PLoS Biology (IF : 8.668)、Environmental Health Perspectives （IF : 8.443）、eLife（IF : 8.303）（图 $8)$ 。

去年排在 21 位和 23 位 的 


\section{Journals not included}

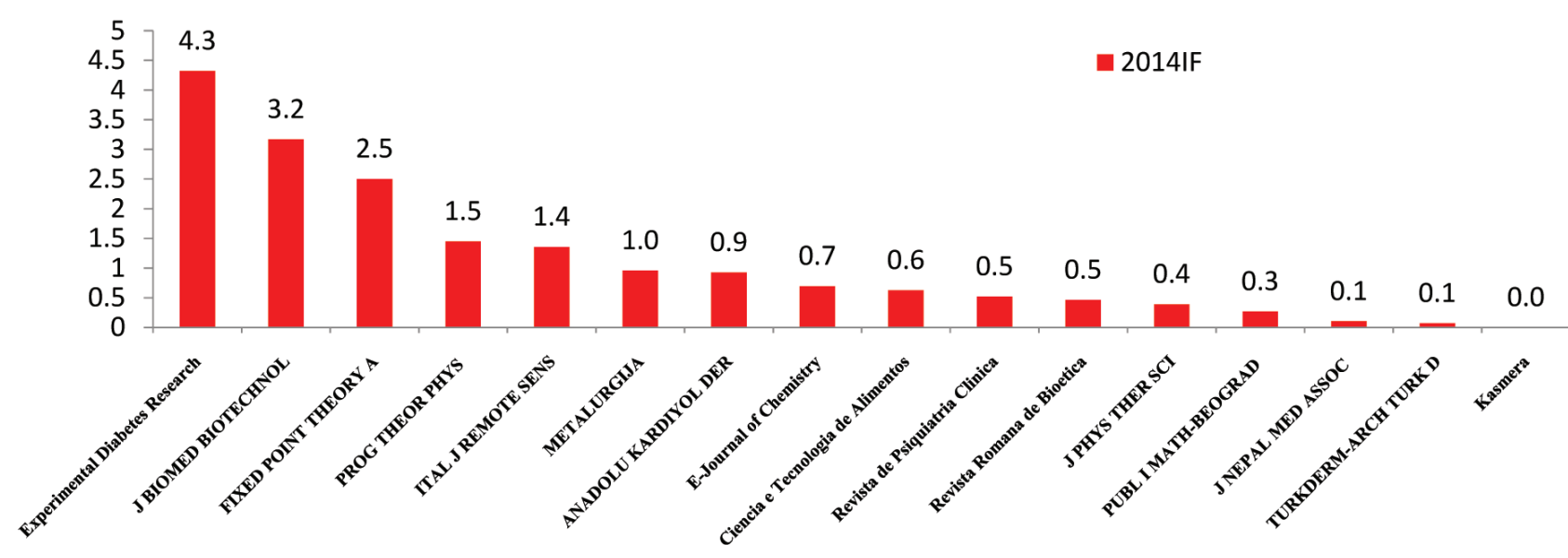

图7. 2016年㿭 “踢出”SCIE的OA期刊

BMC Medicine (IF : 8.005) 和 10.588) 和 Earth System Science increase 之列。

Particle and Fibre Toxicology (IF : Data (IF : 8.286), 它们的第一 8.649) 分别排在今年的第 20 位 年影响因子都不低。另外 Lancet 和 16 位。

Global Health 杂志去年被 SCIE

六、SCIE的OA期刊 In-

creased Top20和De-

新的 Top20 杂志中有 2 份 收录, 今年是被收录的第二年, creased Top20分析

是新收录杂志:Mmwr-Morbidity 与去年的影响因子 10.042 相比, and Mortality Weekly Report (IF : 今年增加了 4.7, 位于 Top20 中

Increased Top20：分析这

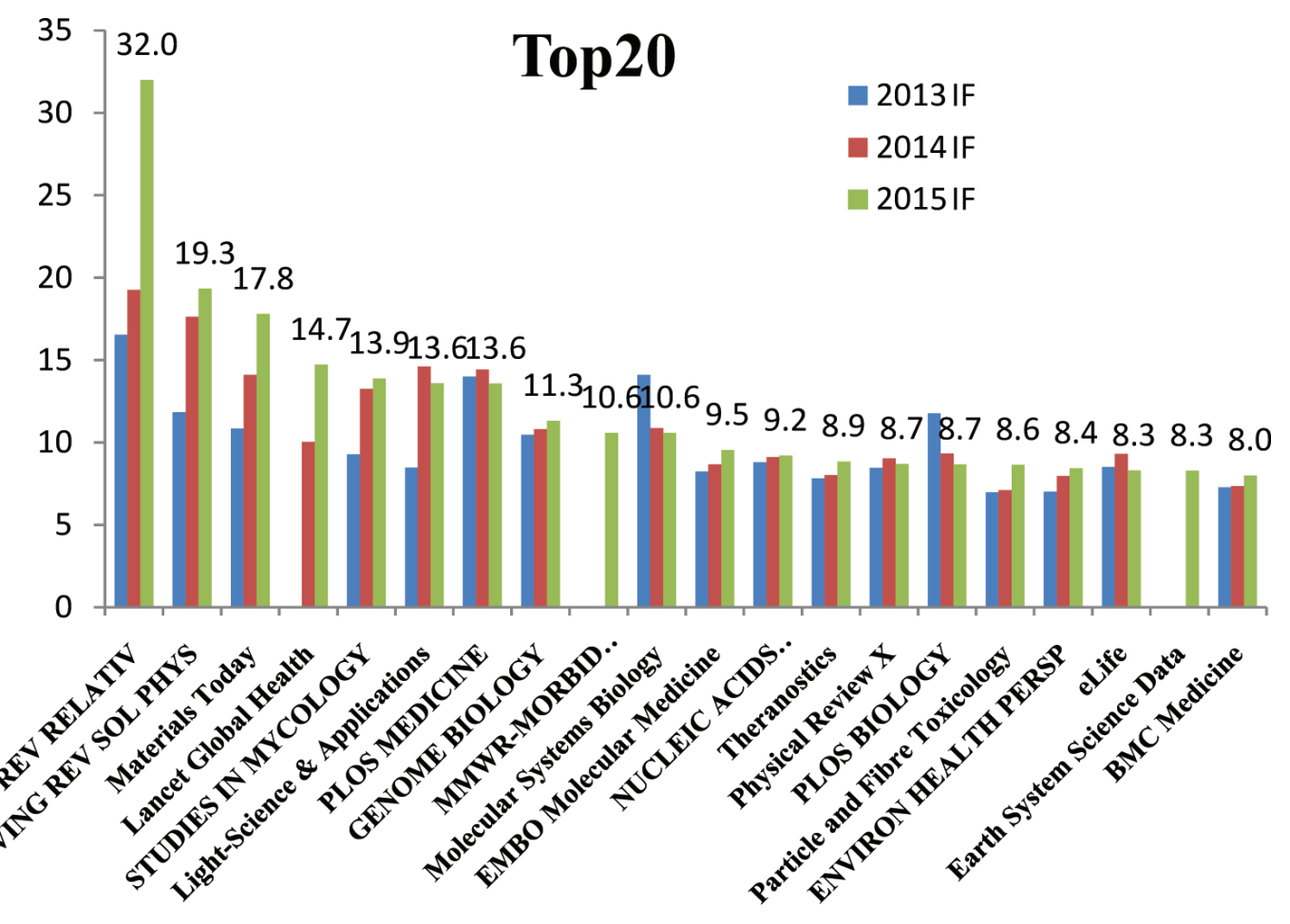

图8.2016年SCIE中Top20的OA期刊 


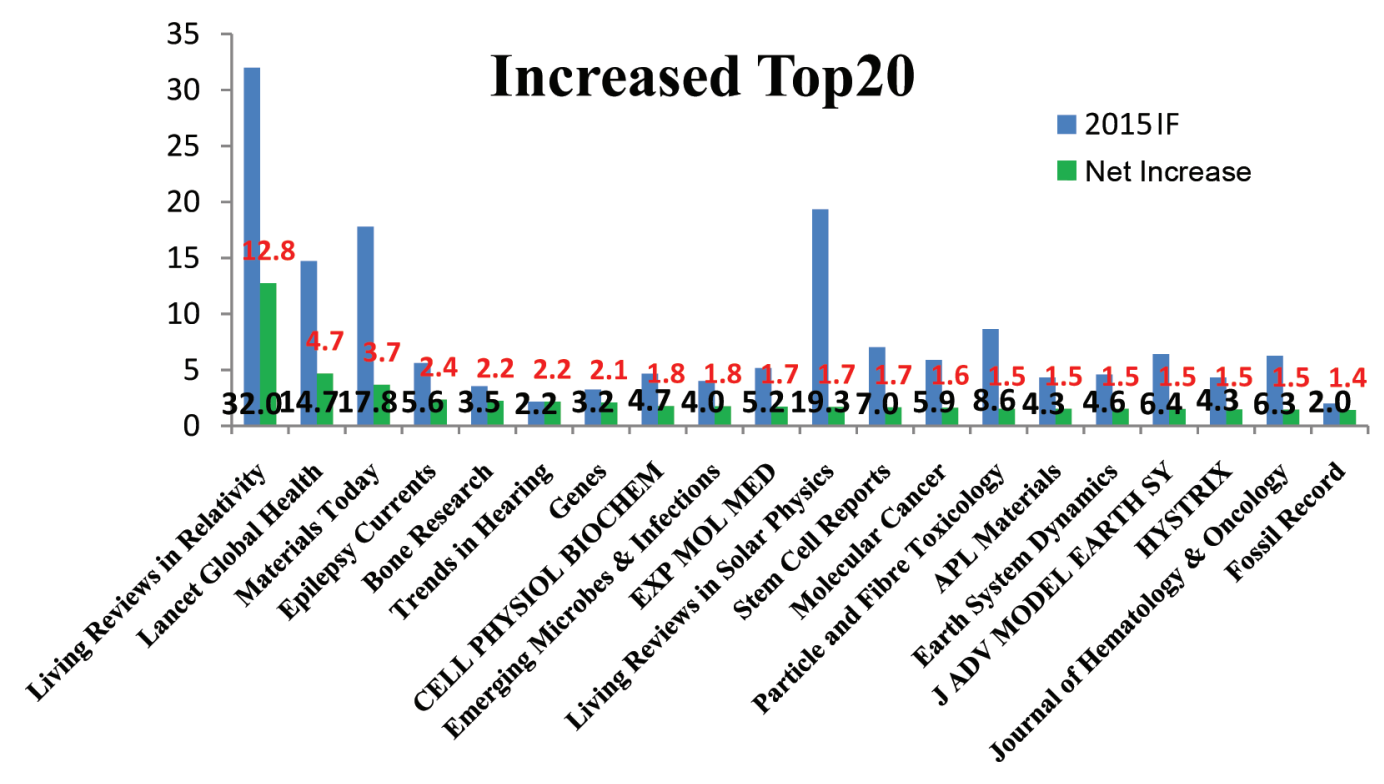

图9. 2016年SCIE的OA期刊Increased Top20分析

1069 杂志中影响因子增加最多的

Top20 杂志发现, 有 7 份杂志 IF 增加了 2 分以上。它们分别为: 增加最多的 Living Reviews in Relativity 杂志, 其 IF 比去年增 加了 12.8 ; 其次是 Lancet Global Health 杂志, 其影响因子比去年 增加了 4.7 ; Materials Today 比去 年增加了 3.7 ;Epilepsy Currents 比去年增加了 $2.4 ;$ Bone Research 和 Trends in Hearing 都比去年 增加了 2.2 ; MDPI AG 旗下的
Genes 杂志由去年的 1.151 上升到 今年的 3.2 , 增加了 2.1 。其余 13 份杂志的 IF 增加了 1.4-1.8(图 9)。

Decreased Top20: 分析这 1069 杂志中影响因子降低最多的 Top20 杂志发现, 有 3 份杂志 IF 降低超过了 2 分以上, 分别为: 降分最多的 Medicine 杂志, 从去 年的 5.723 下降到今年的 1.206 , 下降了 4.5 分; Current Issues in Molecular Biology (CURR ISSUES MOL BIOL）下降了 2.7 分,
从去年的 5.75 下降到今年的 3.083 ; Infectious Diseases of Poverty下降 了 2 分, 从去年的 4.111 下降到今 年的 2.130 。其余 17 份杂志 IF下 降了 2 分以下, 其中 4 份杂志的今 年的 IF 都在 5 分以上 (图 10)。

像这几份相对高分的杂志, 其 IF 降低 1 分多, 笔者认为这算 是正常浮动。至于 IF 降幅巨大的 杂志, 大家肯定特别关心其原因, 比如 Medicine 杂志。该杂志去年 5.723 的影响因子是根据 2012 和

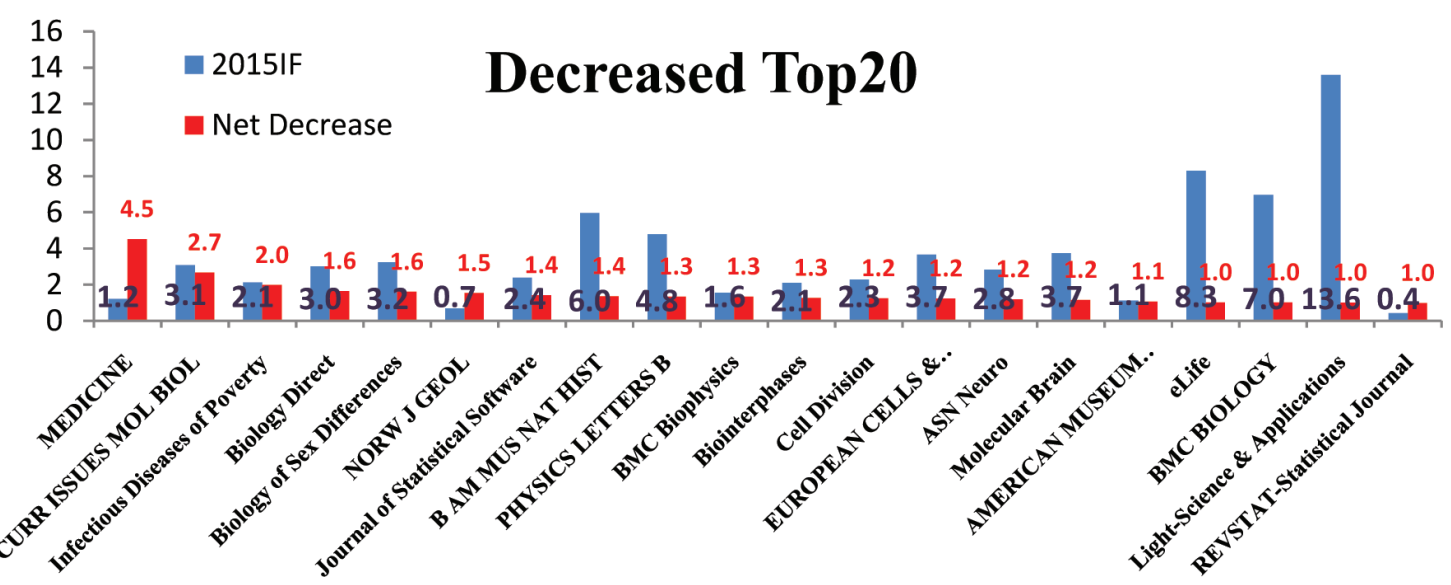

图10.2016年SCIE的OA期刊Decreased Top20分析 


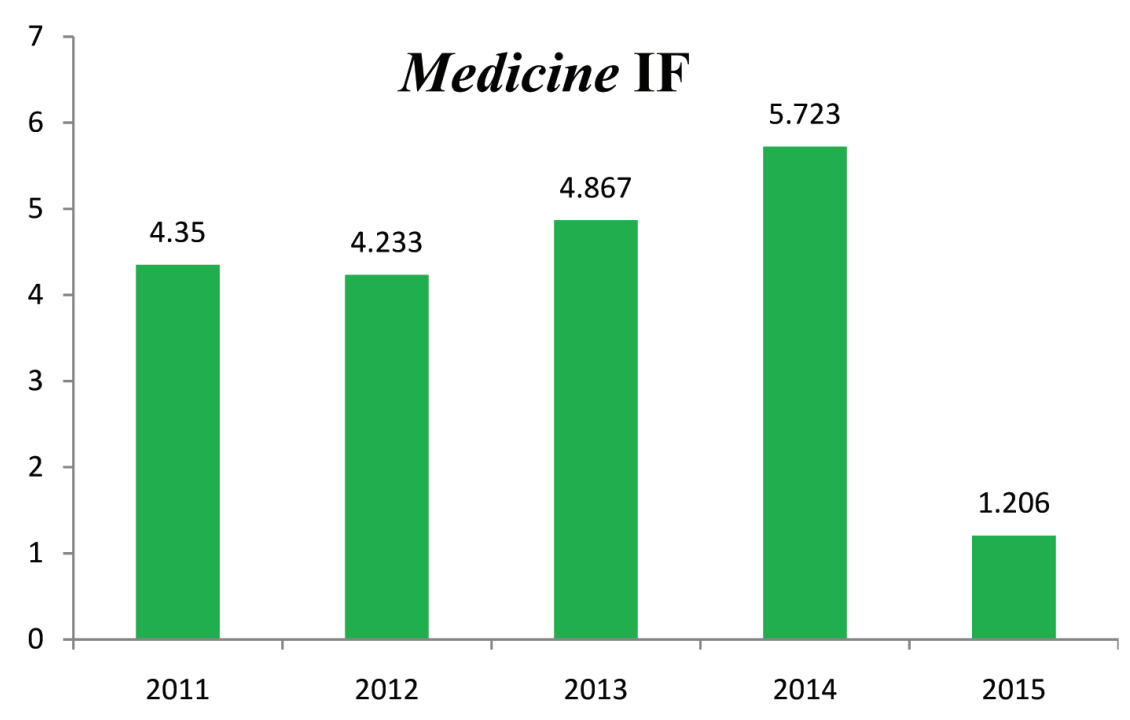

图11.2011-2015 Medicine的影响因子

2013 年所发表的文章数 (35 篇和 30 篇)、及这些文章在 2014 年的 引用数 (分别为 260 和 112) 来计 算的, 为总引用数 372 除以总文 章数 65 得出 5.723 的影响因子。 而该杂志在 2013 年和 2014 年分 别发表了 30 和 315 篇文章, 这两 年文章在 2015 年的引用数分别 为 133 次和 283 次, 所以这两年 的总引用数 416 除以总文章数 345 得出该杂志今年的 1.206 的影响 因子。该杂志 2014 年文章数是其
之前每年发表数的 10 倍, 而引用 数没有相应地增加, 所以导致 IF 剧降。据 Web of Science 数据库 显示, 该杂志 2015 年发布了 1989 篇论文, 如果引用数没有同比增 长, 该杂志明年的影响因子很有 可能会再次降低 (图 11 和图 12)。

\section{Current Issues in Molecular}

Biology 杂志过去 5 年 (2011-2015) 每年发表 6 篇文章。2012 和 2013 这两年的文章在 2014 年的引用数 为 38 和 31 , 从而得出其去年的
5.750 的 IF; 而 2013 和 2014 这两 年的文章, 在 2015 年的引用数为 26 和 11 , 所以总引用数 37 除以总 文章数 12 得出今年的 3.083 的 IF。 该杂志 IF 的降低不是像 Medicine 杂志那样由于文章数剧增导致的, 而是其文章引用数的浮动所致。

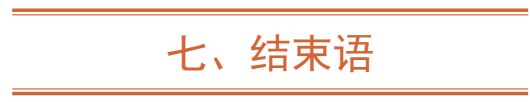

$\mathrm{OA}$ 期刊的出现及发展已 经严重冲击了传统期刊的办刊模

\section{Medicine Papers}

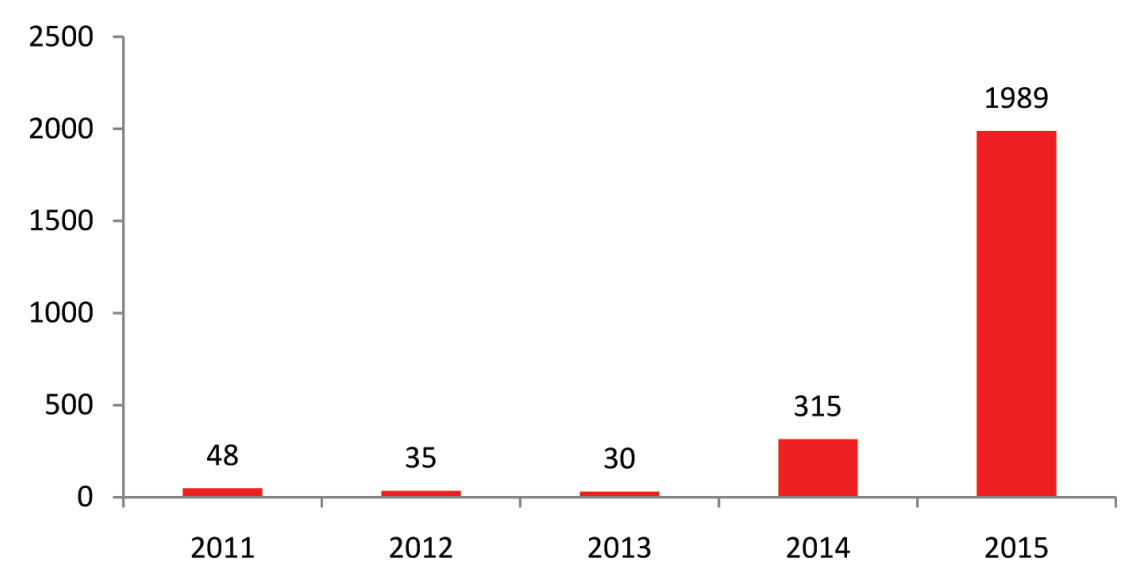

图12.2011-2015年Medicine发表的文章数 
式。 $\mathrm{OA}$ 让读者更能及时把握最 新研究进展, 同时增加了 $\mathrm{OA}$ 期 刊文章的引用。另外, 大家都关 心 OA 期刊的收费问题。绝大多 数 OA 期刊都收取不低的发表费 用 (比如 Genome Biology 的发表 费为 $\$ 2975$, BMC Medicine 的发 表费为 $\$ 2785$, Scientific Reports 的发表费为 $\$ 1,495$, Medicine 的 发表费为 $\$ 1400, \operatorname{PLoS}$ 旗下 7 个 OA 期刊都收费, 见 Table2)。也 有极少数有资金支持的 $\mathrm{OA}$ 期
刊不收费用, 比如美国疾病控制 中心主办的 $\mathrm{OA}$ 期刊 Emerging Infectious Diseases (IF : 6.994, 355 篇/2015 年) 和 Morbidity and Mortality Weekly Report (IF: $10.588,278$ 篇 $/ 2015$ 年) 都是免 费发表, 因为它们有美国联邦政 府的支持; eLife (IF : 8.303, 408 篇/2015 年) 杂志得到美国马里 兰州霍华德・休斯医学研究所、 英国伦敦惠康信托基金会以及德 国柏林马克斯 - 普朗克学会赞助,
目前是免费发表的, 但如果没有 资金支持，可能也会转为收费的。 随着 OA 期刊的增加和竞争的激 烈, 为了争取好的稿源, 将来 $\mathrm{OA}$ 期刊的费用有可能会降低。

\section{参考文献}

[1] Data were accessed and downloaded from InCites Journal Citation Reports of Web of Science.

\section{( 上接第 189 页 )}

细胞周期、细胞凋亡、肿瘤、细 胞信号转导方面的文章, 该刊 的发文量很大, 每年都是将近 1000 篇文章, IF 近几年一直稳 定在 5 分左右, 虽然目前国内关 注的较少, 但是其在国外影响力
还是不错的, 因此是该领域投稿 的好选择。该杂志审稿效率非常 高, 对稿件要求比较严谨, 尤 其是对语言和结构。要求逻辑比 较清楚, 完整性也有较高要求, 而创新性的要求一般。根据其文
章质量, 预测会在近几年再度 冲上 5 分并稳定在 5 分以上。所 以在投稿前, 最好对文章内容和 语言进行比较好的把关, 否则有 可能会以文稿结构和语言被直接 拒稿哦 